\title{
Consensus in Medicine
}

\section{Cervical cancer screening: the Pap smear}

\author{
SUMMARY OF AN NIH CONSENSUS STATEMENT
}

A consensus development conference on cervical cancer screening: the Pap smear, was held at the National Institutes of Health on 23-25 July 1980. Its purpose was to examine the scientific basis for screening for cervical cancer and to make recommendations for the medical community and the public on the use of the Pap smear in screening for cancer of the uterine cervix. No attempt was made to provide recommendations related to screening by the Pap smear for other cancers of the female genital tract-that is, endometrial or ovarian cancers.

Recommendations were based on the accepted definition that screening procedures apply to healthy female populations and not to women identified as patients undergoing gynaecological treatment. The panel considered the questions presented below.

The members of the consensus development panel included epidemiologists, pathologists, obstetricians-gynaecologists, practising physicians, health scientists, a social worker, a lawyer, and consumer representatives.

As a framework for developing recommendations, the panel agreed to the following operational definitions:

- A Pap smear, developed by Dr George N Papanicolaou more than 50 years ago, is a cellular specimen removed from the lower female genital tract which is used in routine screening for cancer. The Pap smear for detecting cervical cancer should include samples of cells from the ectocervix, transformation zone, and endocervical canal.

- In the United States most Pap smears are obtained as part of a medical examination by a woman's doctor or in the context of a health maintenance programme. A cervical cancer screening programme refers to the testing of large numbers of women who show no symptoms of pathological changes of the genital tract in order to classify them as likely or unlikely to have cervical cancer or its precursors.

The conference was sponsored by the National Cancer Institute, the National Institute on Aging, and the National Institute of Child Health and Human Development, in conjunction with the National Center for Health Care Technology and with the help of the Office for Medical Applications of Research, NIH.

Members of the consensus development panel were: Dr Maureen M Henderson, (chairman) University of Washington Health Sciences Center, Seattle, Washington; Dr Katherine F Carson, San Diego, California; Dr Pelayo Correa, Louisiana State University Medical Center, New Orleans, Louisiana; Ms Ellen Flannery, Washington, DC; Dr John K Frost, Johns Hopkins University School of Medicine, Baltimore, Maryland; Ms Genevieve Hill, Atlanta University, Atlanta, Georgia; Dr Gerry B Bill, Cross Cancer Institute, Edmonton, Alberta; Dr George B Hutchison, Harvard School of Public Health, Boston, Massachusetts; Dr Raymond H Kaufman, Baylor College of Medicine, Houston, Texas; Dr John J Mikuta, University of Pennsylvania, Philadelphia, Pennsylvania; Dr Duncan vB Neuhauser, Case Western Reserve University School of Medicine, Cleveland, Ohio; Dr Kenneth L Noller, Mayo Clinic, Rochester, Minnesota; Dr Estelle Ramey, Georgetown University School of Medicine, Washington, DC; Dr Beverly J Williams, University of Tennessee Center for Health Sciences, Memphis, Tennessee. Dr Ralph M Richart, Columbia University, served as a consultant to the panel in the discussion of the presentations. The conference co-ordinators were: Dr Diane J Fink, National Cancer Institute, Bethesda, Maryland; Dr Philip C Prorok, National Cancer Institute, Bethesda, Maryland.
In 1980 the incidence of invasive cervical cancer in the United States is estimated to be 16000 and that of carcinoma in situ, or preinvasive cancer, 45000 . The latest estimate of mortality in 1980 is $7400 .^{1}$

Three characteristics of a disease have been cited as requirements for making it suitable for screening. Firstly, the disease should have serious consequences. Secondly, the disease must have a treatment which, when applied to the screening-detected stage of the disease, is more effective than treatment applied after symptoms have led to a diagnosis. Thirdly, the detectable preclinical phase of the disease should have a high prevalence; otherwise, too few cases would be detected to justify the expense of a screening programme. Cancer of the cervix was considered by this conference in the context of these criteria and reaffirmed as being a disease highly suitable for screening.

(1) Does screening with a Pap smear affect the mortality from cervical cancer?

Evidence suggests that the incidence of invasive squamouscell carcinoma* is falling and mortality from cervical carcinoma is decreasing. At the same time, carcinoma in situ is being detected with increased frequency. These trends have been noted in association with increased screening for cervical carcinoma and are probably related to early histological diagnosis of cervical neoplasia (abnormal cell growth) in verification of the results of cytological screening.

\section{(2) Is the Pap smear safe as a screening procedure?}

There are no known adverse effects that have been ascribed to the Pap smear screening technique. If the Pap smear is incorrectly evaluated by the laboratory or is incorrectly responded to by the clinician, however, over-interpretation may result in unnecessary procedures and in possible complications. $\dagger$

(3a) On the assumption that screening is beneficial should the Pap smear be used as a routine screening procedure?

The panel agreed that the Pap smear should be used as a routine screening procedure for cervical cancer.

\section{(3b) In whom and how often should routine screening} be performed?

At what age should screening become a regular part of a woman's health evaluation?

- At what age is screening no longer rewarding ?

- What is the optimal screening frequency at different ages?

* Most invasive cancers occur now in women who have not been screened at all, rather than in those screened too infrequently.

† Surgical conisation for elucidation of minor changes in Pap smear cytology can occasionally result in severe haemorrhage or in an incompetent or obstructed cervix. 
Should the recommendations made in response to the questions above be modified for certain high- or low-risk groups?

- If so, what are the groups and what are the modifications?

For many years, it has been the policy of health care providers in the United States to recommend annual Pap smear screening for women aged over 18 years. This policy has not been based on the results of well-designed experimental studies, because there have been no rigorous randomised trials of the impact of Pap smear screening on mortality. Nevertheless, this policy has contributed to a reduction in the ill effects and mortality caused by invasive cervical carcinoma. The panel carefully reviewed the data presented at the consensus development conference to determine whether changes in this general screening policy should be suggested. On the basis of available scientific data and judgment, the panel made the following recommendations for screening asymptomatic women:

- Virgins need not be screened for cervical cancer.*

- All women who have had sexual intercourse should be screened for cervical cancer.

(1) Screening should be started soon after the beginning of sexual activity.

(2) If the first smear is satisfactory and does not indicate evidence of neoplasia, the smear should be repeated after one year.

(3) If the second smear is also satisfactory and negative, rescreening of most healthy women should be repeated at regular intervals of one to three years. The decision on precisely how often to rescreen them should be made jointly by the women and their medical care providers. The panel did not agree on precisely how often these examinations should be repeated for healthy women of different ages. For healthy women with different assessed risks of developing cervical cancer, the panel considered that, in general, the level of risk should not determine the frequency of screening.

The assessed risk of a healthy woman getting cervical cancer in the future should play a part in decisions about the frequency of repeated screening only when that assessed risk is either extremely high or extremely low. Any healthy woman whose risk level is assessed as being at or below the level found in the general population is considered to have a low risk level. Any healthy woman whose risk level is assessed as being five times higher than the risk level found in the general population would be considered to have an extremely high risk level, but exclusive application of the factors determining high risk mentioned in the next paragraph has so far not shown any groups of healthy women with a risk level this high. Nevertheless, the level of risk should be the criterion used in identifying groups of women for whom intensive and complete recruitment into screening programmes is critical.

(4) The level of risk referred to in the above paragraph is determined by four factors, three of which are consistently identified with a higher than average likelihood of developing cervical cancer in situ, and the fourth of which is consistently identified with almost complete absence of risk of developing invasive squamous-cell cervical cancer. The factors determining high risk are: first intercourse before 18 years of age; a variety of sex partners; and low socioeconomic status. The factor determining low risk is life-long abstinence from sexual intercourse ("virgin" status).

- If two negative Pap smears are obtained after the woman reaches the age of 60 further screening for cervical cancer appears to be unrewarding. The panel made no assessment of the usefulness of screening in detecting vaginal, endometrial (pertaining to the lining of the uterus), or ovarian cancer.

- A woman whose Pap smear shows significant epithelial abnormality should be referred for diagnostic evaluation as described in the answer to question 5.

*There are some notable exceptions, such as women exposed to stilboestrol in utero, who may later develop adenocarcinomas of the cervix and vagina.
Unscreened women at risk should be recruited into the screening programmes. Recruitment could be aimed at points of contact with medical care, such as neighbourhood clinics, emergency rooms, venereal disease clinics, sites of immigration examinations, jails, hospitals, and all doctors' offices. Evidence suggests that education on the high risk factors for cervical cancer would increase the use of screening.

- A randomised clinical trial to determine optimal screening intervals is not recommended.

\section{(4) What critical factors are needed to ensure that the} procedure is reliable?

Key factors affecting reliability include a proper clinical sample, high-quality laboratory evaluation, and proper communication between the cytopathologist and the clinician.

The quality of a laboratory's cytopathological technique is of prime importance to any screening programme, but high quality is difficult and expensive to attain and to maintain. While certain technical points can be evaluated by federal agencies (such as the Center for Disease Control) the key factors in ensuring consistently good cytopathological practice are the qualifications and continuing education of the staff as well as the quality assurance programme of the laboratory. Quality is best assured if a laboratory meets the accreditation standards of the American Society of Cytology and the College of American Pathologists. There must be adequate staffing to maintain high quality. The standards of the American Society of Cytology (currently a maximum work load per cytotechnologist of 12000 cases per year for screening) and those recommended in the Canadian Walton Report ${ }^{2}$ (currently, three cytotechnologists per 25000 cases per year for screening, quality assurance, supervision, etc) are recommended. The technical staff must be supported by adequate clerical staff and staff proficient in cytopreparatory techniques.

Accurate and complete reporting between the cytology laboratory and the clinician is of basic importance to proper Pap screening. Poor wording of the report and the use of laboratories at great distances from the clinician can impair proper communication. The use of numerical classification, such as Pap $\mathrm{I}-\mathrm{V}$, in place of diagnostic terminology is discouraged and should be abandoned in favour of acceptable, standard, clearly understood medical disease nomenclature. Neoplasia of the cervix develops as a progressive range of tissue changes which terminate in lethal invasive carcinoma, with exfoliated (shed) cells showing characteristic neoplastic changes. Any specific changes detected by Pap smear screening are best stated in clear, mutually understood, diagnostic terms. In this way the biological significance and recommendations for diagnostic investigation and handling are reported completely and clearly to the clinician, so that the severity of the changes is understood, and the clinician has clear guidance for appropriate action.

\section{(5) Following screening what are the responsibilities for} follow-up, confirmation of findings, and initiation of treatment?

The panel recommended that whenever a clinician receives a Pap smear report that suggests cervical neoplasia (dysplasia or cellular anomalies; carcinoma in situ; invasive cancer) the patient must undergo thorough diagnostic evaluation. It is the responsibility of the physician, or his designated substitute, to notify the patient of the abnormal result. The objective of the diagnostic investigation is to use the simplest procedure to ensure an accurate diagnosis.

Ideally, diagnostic evaluation should include colposcopic examination and biopsies of representative areas of the cervix, usually including an endocervical curettage (scraping of the 
interior wall of the cervix). Diagnostic conisation of the cervix (removal of a cone of tissue) may also be required if the location of the lesion makes colposcopy unsatisfactory, if the diagnosis from an endocervical curettage so indicates, or if the cytological and histological findings conflict.

Treatment of neoplastic lesions must depend on the individual case. In certain cases non-invasive lesions may be treated in outpatient departments where staff have proper skills in gynaecological oncology. On the other hand, invasive cervical carcinoma requires the use of hospital facilities in addition to or consultation with a doctor with skill in gynaecological oncology. The condition of the woman who has been treated for cervical neoplasia should be closely followed.

\section{Further investigations}

The crucial unanswered question is whether carcinoma in situ develops and progresses at different rates in women with different assessed levels of risk. The panel therefore recommended research on the rate of progression of presymptomatic disease in high-risk women. The panel also recommended that studies be undertaken to monitor the impact of changes in the Pap smear screening interval on the incidence and mortality rates of cervical carcinoma. Finally, it recommended that a task force be convened within five years to assess all relevant scientific data that may have accumulated since July 1980 .

\section{Minority opinion}

Review of the several computer-based, cost-effective decision models does not justify yearly screening unless the risk ratio for high-risk groups is at least 5.0. In the absence of such an increased risk after two negative tests, screening intervals greater than three years could be appropriate. In my opinion the probability that screening prolongs life ranges from 0.50 to $\geqslant 0.99$. These decision models are sensitive to the lower end of this probability range only if the screening interval is long. Therefore a randomised clinical trial does not seem justified. Computer-based models should be widely available in interactive form allowing providers and patients to vary the assumptions and consider the results. These models should be used in high school courses and in health science museums. On the surface the controversial issue is the frequency of screening. The real issue, however, is the role of computer models in clinical decision making. These powerful techniques are new to medicine, and not enough doctors are being educated in their use. This Pap smear debate will be viewed as one of the historical landmarks in this transformation in clinical reasoning.-DUNCAN NEUHAUSER, PHD (economics).

\section{References}

1 Silverberg E. Cancer statistics 1980. Cancer fournal for Clinicians 1980; $30: 23-44$.

2 Task Force. Cervical cancer screening programs. Can Med Assoc $\mathcal{F} 1976$; 114:1003-33.

\section{MATERIA NON MEDICA}

\section{Living - on cloud nine}

By a longstanding arrangement, our practice provides the police surgeons for the winter months. As general practitioners in a small country town we usually find it a quiet addition to our work, though tedious in the middle of the night. Once or twice a week we collect blood samples from drinking drivers and occasionally we certify death in a gruesome road accident.

The strike of prison officers has altered all that. Our local nick is now a temporary gaol for remand prisoners, as my partner discovered when, asked to drop in on his way home, he was faced with 17 newly arrived prisoners to examine. My turn came next morning.

By this time, I had read of the recent hardships at Nottingham and felt disturbed and sympathetic for our very polyglot visitors, packed three to a cell with none of the usual facilities of a remand prison. Perhaps my concern showed in my face. The prisoners, whose minor complaints were clearly an excuse to break the monotony by sampling the local doctors, were suspicious at first and effusively courteous later. Proper gentlemen they were, one and all. More consultations followed over the weekend and again today; filling in the attendance forms is our hardest job. But my concern was misplaced. They love it here.

The police station is a modern building with reasonable cells and a small exercise yard, forming a very secure complex, within which our guests have a limited freedom. They are not locked away all day. Meals come in regularly from the pub next door, which is delighted at this sudden bonus of customers now the holiday season is over. There is a colour television; two prisoners are painting a large Cinderella's coach for a children's party, while two more are engrossed on the floor with a large jigsaw puzzle. A muscular West Indian, anxious to please, is beginning to bore with his frequent demonstrations of press-ups and hand-stands on one hand. Others play a version of the Eton wall game in the yard. One from Eastern Europe claims to have been his country's top pop singer; he has not sung for us yet, but he will. But most of all they think our police are wonderful.

"It's like this," said a constable. "We've got nothing against them, we haven't nicked them. They are villains, of course, but if they're all right with us we are with them."

Our local police are, in fact, a nice lot. You would be foolish to push your luck with them; they can be tough if required and are no fools, but their instincts are kindly. One prisoner, tears in his eyes, said he'd "never been treated so nice"; he didn't know police could be so kind.
All of them combined to write a letter to "the Super" expressing the hope they could come back here again "afterwards."

"I sincerely hope not," said the Super, viewing a corner of his station that was looking more and more like a holiday camp. One prisoner, careful not to cheat the post office, posted a letter addressed to "the Governor" with a 12 p stamp on it through the grille of his cell to say he had loved every moment of the weekend to the full: "On cloud nine; never a dull moment." At the bottom he wrote the word "living!" and circled it. It was pathetic as well as comic. Film this for a comedy, I thought, and they would say you had overdone it.

I removed some stitches from a cockney's finger. He had cut it while in Belgium. The hotel had called a doctor, who put in four stitches.

"Charged me twenty quid, he did; bloody daylight robbery if you ask me." We agreed, vehemently. It was a wicked world, and I kept a straight face.

As for our police, in their unaccustomed role of prison officers, overtime has rocketed. "Comes in handy before Christmas," said one. "You can say that again." It can't last long, but while it does our guests are possibly learning some new tolerance of authority, a lesson born of kindness; although some, I have no doubt, would still nick my car or wallet if they got the chance, and be puzzled if I looked hurt. The police are looking strangely content-at the moment anyhowand the pub next door is certainly smiling. As for our practice, the partners are filling in the claim forms for payment, one for each consultation. So far it is more than 30 in five days. I have a feeling that, taking the country as a whole, this is going to be a very expensive strike with unexpected consequences.-POLICE SURGEON.

When is it safe to resume sexual intercourse without fear of pregnancy after the menopause?

The menopause is the cessation of menstruation. Ovulation may occur sporadically for a little time after the menopause. This is relatively uncommon in women of this age group, especially if there are also hot flushes, which denote low oestrogen concentrations and therefore probably no ripening Graafian follicles. If there are no periods for a year then almost certainly ovulation will have ceased. A barrier contraceptive might be most appropriate during this time. Steroid hormone pills confuse the issue by causing uterine bleeding. 